ARTICLE

\title{
Stereoselective gridization and polygridization with centrosymmetric molecular packing
}

Dongqing Lin ${ }^{1,4}$, Ying Wei ${ }^{1,4}$, Aizhong Peng ${ }^{1}$, He Zhang ${ }^{1}$, Chunxiao Zhong ${ }^{1}$, Dan Lu ${ }^{2}$, Hao Zhang ${ }^{2}$,

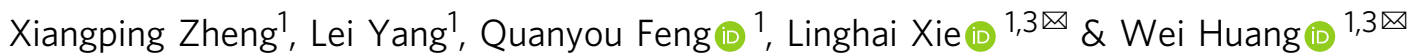

The gridarenes, with well-defined edges and vertices, represent versatile nanoscale building blocks for the installation of frameworks and architectures but suffer from difficulty in stereoselective control during their synthesis. Here we report a diastereoselective gridization of superelectrophilic diazafluorene-containing substrates $\left(A_{m} B_{n}\right)$ with crescent shapes into Drawing Hands grids (DHGs). The meso-selectivity reaches $75.6 \%$ diastereomeric excess (de) during the gridization of $A_{1} B_{1}$-type substrates and maintains $\sim 80 \%$ de during the polygridization of $A_{2} B_{2}$-type monomers. Such stereocontrol originates from the centrosymmetric molecular packing of two charge-delocalized superelectrophiles with synergistically $\pi-\pi$ stacking attractions and coulombic repulsions. As meso-stereoregular structures show $20 \sim 30 \mathrm{~nm}$ in length, the rigid ring/chain-alternating polygrids have a Mark-Houwink exponent of 1.651 and a molecular weight $(M)$ dependence of the hydrodynamic radius $R_{\mathrm{h}} \sim M^{1.13}$. Via the simulation of chain collapse, meso-configured polygridarenes still adopt rod-like conformations that facilitate the high rigidity of organic nanopolymers, distinguished from toroid backbones of rac-type polygrids.

\footnotetext{
${ }^{1}$ Centre for Molecular Systems and Organic Devices (CMSOD), Key Laboratory for Organic Electronics and Information Displays and Jiangsu Key Laboratory for Biosensors, Institute of Advanced Materials (IAM), Nanjing University of Posts and Telecommunications, 9 Wenyuan Road, Nanjing 210023, China.

${ }^{2}$ State Key Laboratory of Supramolecular Structure and Materials, College of Chemistry, Jilin University, 2699 Qianjin Avenue, Changchun 130012, China.

${ }^{3}$ Shaanxi Institute of Flexible Electronics (SIFE), Northwestern Polytechnical University (NPU), 127 West Youyi Road, Xi'an 710072, China. ${ }^{4}$ These authors

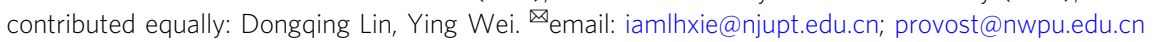


C yclophanes are heteroatom/C $\left(s p^{3}\right)$-hybridized arene macrocycles, which have been transformed into calix $[n]$ arenes ${ }^{1}$, pillar $[n]$ arenes $^{2}$, and three-dimensional (3D) cages $^{3}$ in the application of host-guest recognition ${ }^{4}$ since they were first synthesized in $1949^{5}$. Further, these structures have been interlocked into catenanes ${ }^{6}$, rotaxanes ${ }^{7}$, and knots $^{8}$, as the candidate for molecular machines ${ }^{6}$. However, most of them are difficult to construct nanoarchitectures such as nanorods, frameworks, and nanorings that have been constructed by porphyrin derivatives ${ }^{9,10}$. Inspired by their presence in fullerenes ${ }^{11}$, carbon nanotubes ${ }^{12}$, and warped nanographene ${ }^{13}$, fluorenyl groups were introduced into nanoscaffolds that endow enhanced rigidity, orthogonal backbones, and predetermined extension sites, as well as wide bandgap properties ${ }^{14}$ and molecular motor behaviors ${ }^{15}$. According to grid complexes from Lehn and colleagues ${ }^{16}$, they are called gridarenes ${ }^{17}$ because of well-defined edges and vertexes that afford them extendability, installability, programmability, and scalability. These nanoscale gridarenes with the highest symmetric backbones can be categorized into five types involving $\mathrm{C}_{2}$-symmetric angle-lost types with two extension sites, $\mathrm{C}_{2 \mathrm{v}^{-}}$ symmetric ladder types ${ }^{18}$ with two pairs of parallelly distributed extension sites, $\mathrm{C}_{4}$-symmetric windmill types ${ }^{19}$ with four radial distributed extension sites, $\mathrm{C}_{2}$-symmetric rhombus types with six extension sites, and $\mathrm{C}_{2 \mathrm{v}}$-symmetric tic-tac-toe types ${ }^{20}$ with eight extension sites. However, the $\mathrm{C}\left(s p^{3}\right)$ atom at the 9-position of a fluorenyl moiety serves as a chiral center that results in various stereoisomers during gridization reactions ${ }^{19}$. For example, windmill-like gridarenes ${ }^{19}$ have 6 stereoisomers within 2 pairs of enantiomers, ladder-like grids ${ }^{18}$ have 7 stereoisomers within 2 pairs of enantiomers, and 日-shaped digrid nanoscaffolds ${ }^{21}$ even have 27 stereoisomers within 12 pairs of enantiomers. The tacticity of gridarenes ${ }^{18,20}$ and polygridarenes would potentially affect the crystallinity ${ }^{22}$, exciton behaviors ${ }^{23}$, and drug activity ${ }^{24}$. Therefore, it is significant to control stereoselective synthesis that would benefit for the molecular engineering of state-of-the-art model and materials.

Stereoselectivity arises from asymmetric attack orientations of intermediates, commonly induced by molecular orbital interactions $^{25}$, steric effect ${ }^{26}$, electronic effect ${ }^{26}$, and supramolecular bonds ${ }^{27}$. In detail, the preferred attack orientation originates from the most stable molecular arrangement ${ }^{26}$, which can be dominated by the packing mode of substrates with sufficient noncovalent interactions including hydrogen bonds ${ }^{28}, \pi-\pi$ stacking ${ }^{29}$, and coulombic repulsion $^{30}$. Encouraged by this, we exploit synergistically supramolecular attraction-repulsion interactions ${ }^{31}$ to tentatively control over the stereoselective pathways of carbocation intermediates during the Friedel-Crafts gridizations and polygridizations (gridization-type polymerizations). To eliminate the stoichiometric effect ${ }^{32}$ on gridizations, we design heterodifunctional $\mathrm{A}_{1} \mathrm{~B}_{1}$-type substrates consisting of an alcohol group as the A-part and a benzenoid moiety as the B-part. For the A-part with the same fluorenyl skeleton, we select a diazafluorenyl group (DAF), which is a kind of electron acceptors ${ }^{33}$ with metal coordination $^{34}$, superelectrophile ${ }^{35}$, and hydrogen bonding behaviors ${ }^{36}$. For the B-part, a carbazole moiety $(\mathrm{Cz})$ with a 3-position linked to a DAF group is used to construct mono-crescent-shaped substrates, called MCs. Distinguished from other $\mathrm{A}_{1} \mathrm{~B}_{1}$-type substrates with a thiophene moiety ${ }^{19}$ or a $\mathrm{Cz}$ moiety linked at a 1-/2-/9position, MCs take advantage of geometry matching to form gridarenes with only two chiral centers and minimized strain energy. These gridarenes in angle-lost shape resemble the famous lithograph art of "Drawing Hands"; thus, we coin them Drawing Hands gridarenes (DHGs, in Fig. 1). During the gridization of MC substrates, we unexpectedly observe that most of DHGs exhibit meso-configurations rather than thermodynamic rac-isomers. Moreover, we demonstrate such gridization rule to transform into stereoselective polygridization, as the inverse process of the ringopening polymerization ${ }^{37}$, to afford rigid rod-like polygrids with meso-tacticity.

\section{Results}

Investigation of gridization rules. Before the gridization of $A_{1} B_{1}$-type substrates, we carried out a Friedel-Crafts prototype reaction of diazafluorene-alcohol 1 a with $\mathrm{Cz}$ in a $1: 1$ equivalent ratio (Fig. 2a). Considering the formation of tricationic superelectrophiles ${ }^{35,38}$ on the DAF moieties, we used $\mathrm{CF}_{3} \mathrm{SO}_{3} \mathrm{H}$ (75 equiv) to initiate the reaction that produced monosubstituted 2a in $14 \%$ yield and disubstituted $\mathbf{3 a}$ in $58 \%$ yield but the dehydroxylated byproducts (4a and 5a, in Supplementary Fig. 1) were also generated. Luckily, incorporating a methoxyl group (substrate $\mathbf{1 b}$ ) led to monosubstituted $\mathbf{2 b}$ in $13 \%$ yield and disubstituted $\mathbf{3 b}$ in $85 \%$ yield within $10 \mathrm{~s}$, which reveals the in situ acceleration of the reaction with monosubstituted products. Interestingly, such acceleration was not observed in the reaction system where the fluorenyl substrate $\mathbf{F}-\mathbf{1 b}$ generated disubstituted F-3b in 22\% yield (Supplementary Fig. 2). As electronic effects cannot explain this phenomenon, a more rational explanation is proposed based on intermolecular aggregation via hydrogen bonds between DAF moieties and strong acid molecules ${ }^{39}$. Considering the stronger hydrogen bonds derived from increased acidity $^{40}$, we hypothesized that this aggregation strength could be tuned by Brønsted acid additives before initiating the reaction.
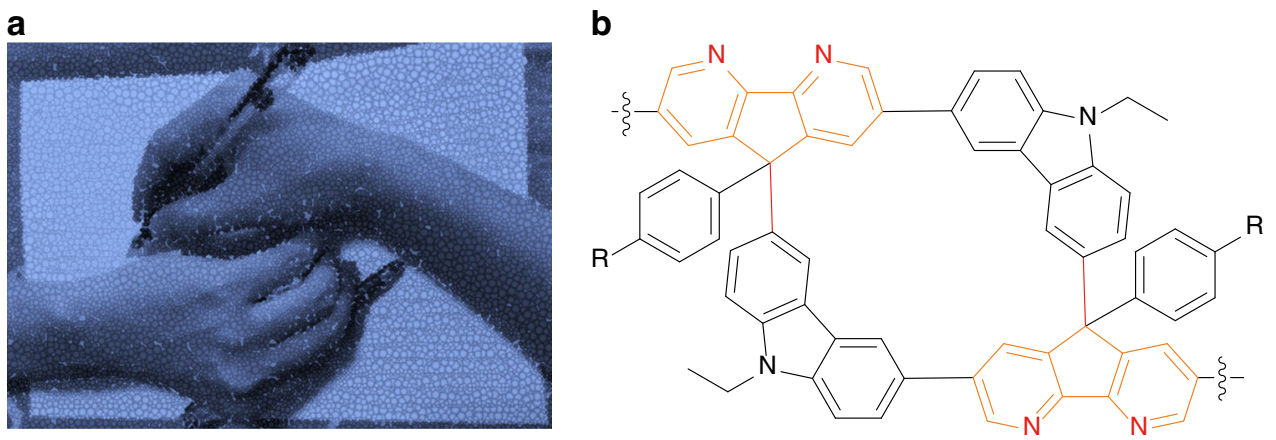

Fig. 1 The conceptual scheme of Drawing Hands art and molecular Drawing Hands gridarene (DHG) structures as a model of the nano-linkage. a The picture of Drawing Hands art created by one of the corresponding author Professor Linghai Xie, without using any element from a database. The ball-point pens are analogous to the $\mathrm{C}\left(s p^{2}\right)-\mathrm{C}\left(s p^{3}\right)$ bonds between the 9-position of diazafluorenyl groups and the 3-position of the carbazole segments. $\mathbf{b}$ The molecular structures of Drawing Hands gridarenes. The orange lines and two nitrogen atoms (in red) depict the diazafluorenyl groups (DAFs). The red lines represent the linkage between the 9-position of diazafluorenyl groups and the 3-position of the carbazole segments, which occurs during the gridization process. 
a

Friedel-Crafts reactions

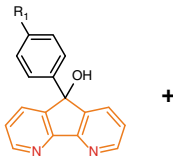

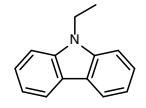

$\mathrm{Cz}$

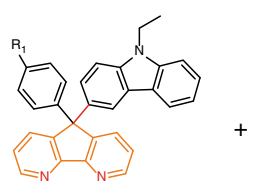

2

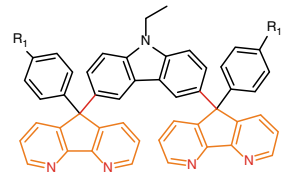

$\mathrm{R}_{1}=\mathrm{H}: 1 \mathrm{a}, 2 \mathrm{a}, 3 \mathrm{a}$

$\mathrm{R}_{1}=\mathrm{OCH}_{3}: 1 \mathrm{~b}, 2 \mathrm{~b}, 3 \mathrm{~b}$

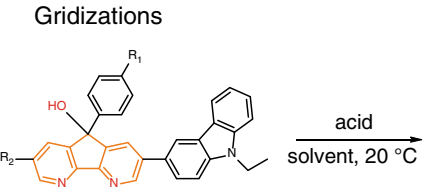

MCs

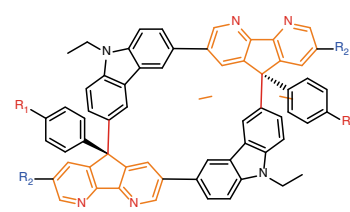

meso-DHGs

$\mathrm{R}_{1}=\mathrm{OCH}_{3}, \mathrm{R}_{2}=\mathrm{H}: \quad \mathrm{MC1}$, meso-DHG1, rac-DHG1, MG1

$\mathrm{R}_{1}=\mathrm{CH}_{3}, \mathrm{R}_{2}=\mathrm{H}: \quad$ MC2, meso-DHG2, rac-DHG2, MG2

$\mathrm{R}_{1}=\mathrm{OCH}_{3}, \mathrm{R}_{2}=\mathrm{Br}: \quad \mathrm{MC} 3$, meso-DHG3, rac-DHG3, MG3

$\mathrm{R}_{1}=\mathrm{OCH}_{3}, \mathrm{R}_{2}=\mathrm{C} 8 \mathrm{~F}:$ MC4, meso-DHG4, rac-DHG4, MG4

$\mathrm{R}_{1}=\mathrm{OCH}_{3}, \mathrm{R}_{2}=\mathrm{SFX}$ : MC5, meso-DHG5, rac-DHG5, MG5

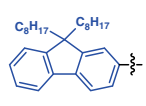

$\mathrm{C8F}$

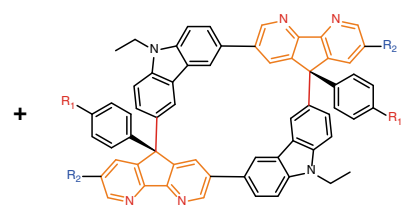

rac-DHGs
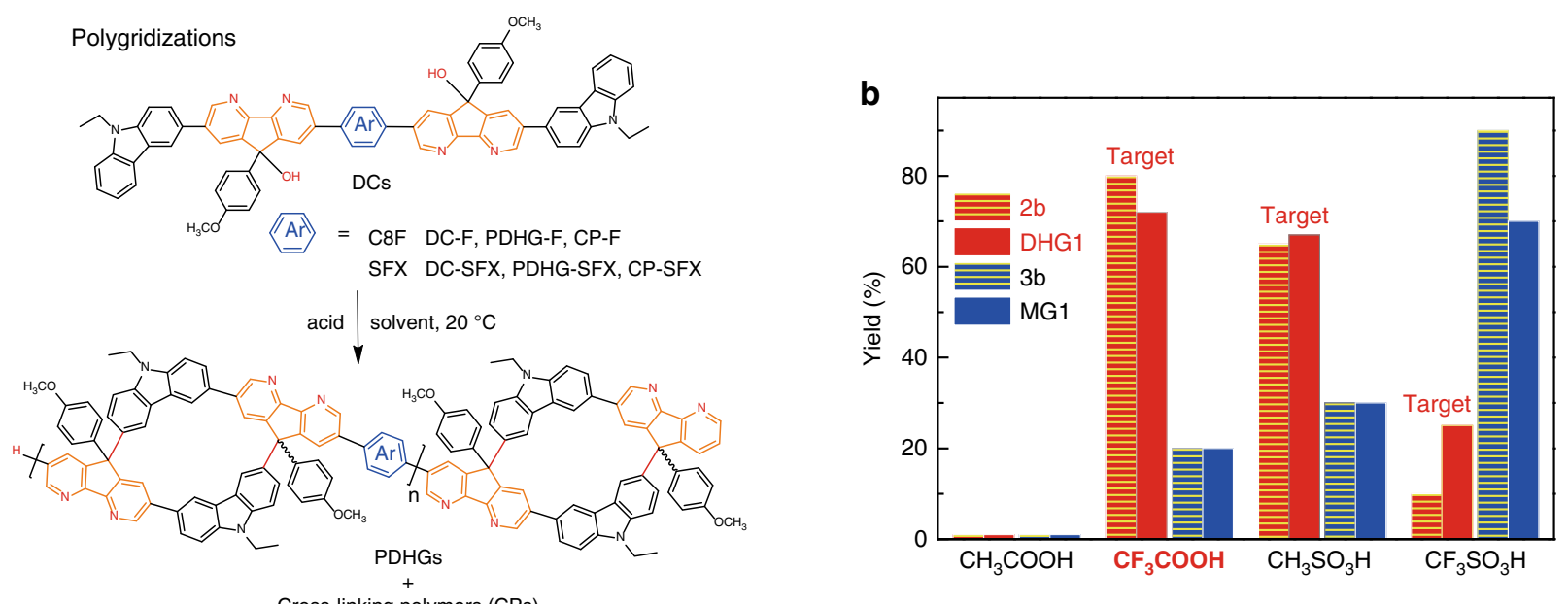

Cross-linking polymers (CPs)
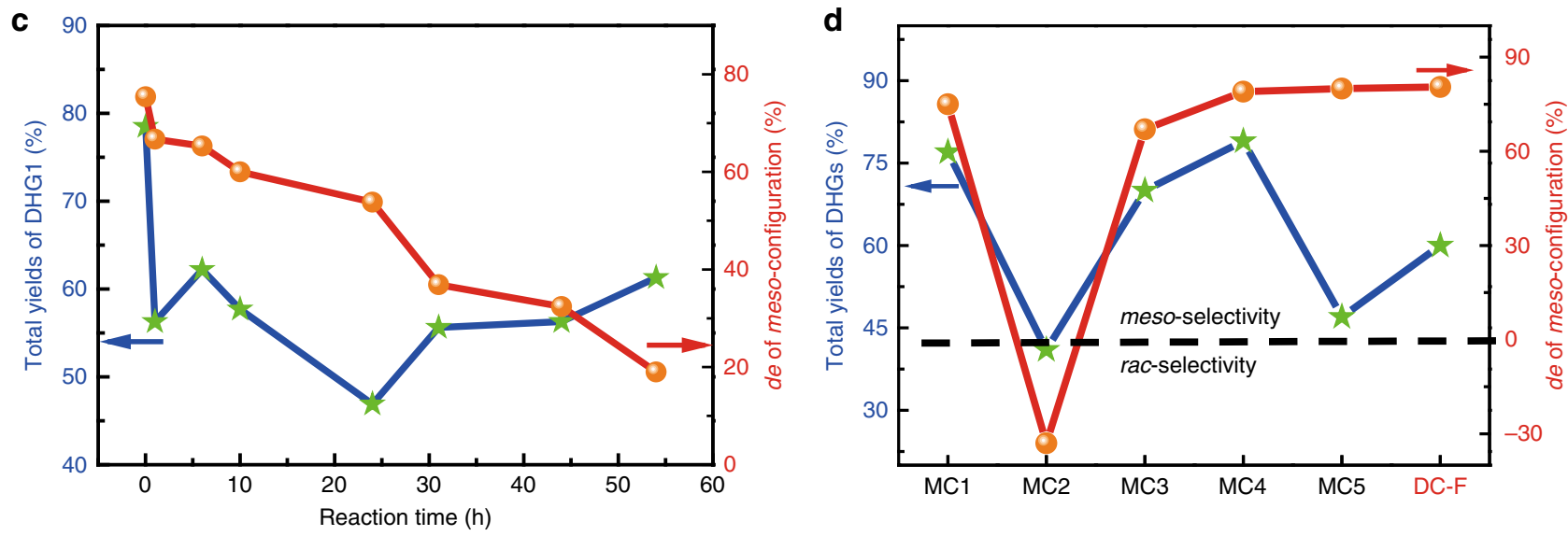

Fig. 2 Characteristics of the Friedel-Crafts reactions, gridizations, and polygridizations. a Corresponding formula. The $R_{1}$ and $R_{2}$ groups are depicted in red and blue, respectively. $\mathbf{b}$ The dependence of acid additives on the yields (after $30 \mathrm{~s}$ of reactions). The volume ratio of the acid additive to dichloromethane (DCM) is about 1/3. The concentration of substrates is about 10 15 mM. For the Friedel-Crafts reactions, the height of red and yellow column corresponds to the yield of $\mathbf{2} \mathbf{b}$; the height of blue and yellow column represents the yield of $\mathbf{3} \mathbf{b}$. For the gridization reactions, the heights of the pure red column and the pure blue column correspond to the yields of DHG1 and MG1, respectively. c The dependence of gridization times on the mesoselectivity of $\mathrm{MC1}$, under the conditions of $\mathrm{DCM}$ solvent, $\mathrm{CF}_{3} \mathrm{COOH}$ acid additive, and $\mathrm{CF}_{3} \mathrm{SO}_{3} \mathrm{H}$ acid catalyst. The blue line and green stars exhibit the total yields of DHG1, and the red line and orange dots show the de value of meso-configuration. $\mathbf{d}$ The total yields (green dot and blue line) and the mesoselectivity of DHG-based products (orange dot and red line) under the optimized conditions in 1 min. The black dashed line is the boundary between the meso-selectivity and rac-selectivity. The yields of PDHG-F is defined as the sample after fully undergoing Soxhlet extraction. 
Consequently, we observed the dependence of $\mathbf{3 b}$ yield on the acidity of the acid additives (Fig. 2b), including $\mathrm{CH}_{3} \mathrm{SO}_{3} \mathrm{H}(35 \%)$, $\mathrm{CF}_{3} \mathrm{COOH}(20 \%)$, and $\mathrm{CH}_{3} \mathrm{COOH}(0 \%)$. These results suggest that the reaction pathways could be flexibly tuned by intermolecular aggregation (reconfirmed by the solvent dependence in Supplementary Fig. 3).

Based on prototype reactions, we investigated the gridization (in Fig. 2a) of substrate MC1 with a methoxyl group that eliminates the generation of dehydroxylated byproducts. However, we observed that the conditions favoring disubstitution $\left(\mathrm{CF}_{3} \mathrm{SO}_{3} \mathrm{H}\right.$ additive, dichloromethane (DCM) solvent) generate target DHG1 in only $8 \%$ yield, whereas they afford macrogridarene oligomers MG1 in $42 \%$ yield. This results suggest that the serious aggregation are unfavorable for the formation of DHG products. In contrast, the moderated aggregation conditions (favoring monosubstitution) afford DHG1 (within $1 \mathrm{~min}$ ) in $68 \%$ yield via the $\mathrm{CH}_{3} \mathrm{SO}_{3} \mathrm{H}$ additive and in $77 \%$ yield via the $\mathrm{CF}_{3} \mathrm{COOH}$ additive (Fig. 2b). More unexpectedly, under the condition of $\mathrm{CF}_{3} \mathrm{COOH}$ additive, we found that the ratio of mesoDHG1:rac-DHG1 was $7.2: 1$, which is identical to $75.6 \%$ diastereomeric excess ( $d e$ ) of the meso-configuration. Considering the thermodynamic equilibrium of Friedel-Crafts reactions ${ }^{41}$, we prolonged the reaction time and found that the total DHG1 yields were overall reduced from $77 \%$ to $55 \%$ (Fig. 2c). Further, the meso-stereoselectivity was reduced from $75.6 \%$ de $(1 \mathrm{~min}), 53.8 \%$ de $(24 \mathrm{~h})$ to $19.0 \%$ de $(54 \mathrm{~h})$, corresponding to the gradually lowered meso-DHG1 yields from $69 \%$ to $36.5 \%$ (Supplementary Fig. 4). These results indicate that the meso-DHG1 is the kinetic product that can transform into MG1. Thus, ultrafast quenching ( $1 \mathrm{~min}$ after the gridization) is key to the meso-selective gridization. However, when using methyl-based MC2 substrates, the meso-selectivity can transform into rac-selectivity (19 26\% de of rac-DHG2) along with negligible gridization time effect (Supplementary Figs. 5 and 6). Hence, the oxygen of a methoxyl group plays a key role in dominating the kineic meso-selective pathways. To further test the scope of diastereoselectivity, MC1 derivatives with various $R_{2}$ groups were reacted under the optimized conditions (Fig. 2d). For example, the Br-containing substrate MC3 affords meso-DHG3 in 61\% yield and $67 \%$ de, indicating that the meso-selectivity cannot be evidently affected by bromine atoms. Furthermore, the meso-stereocontrol is maintained if incorporating $9^{\prime} 9$-dioctylfluorenyl group $\mathrm{C} 8 \mathrm{~F}$ (MC4, in $82 \% d e$ ) or spiro(fluorene-9, $9^{\prime}$-xanthene) group SFX (MC5, in $80 \% d e$ ), although the SFX group decreases the DHG5 yield to $43 \%$. Overall, $\mathrm{R}_{2}$ groups can affect $\mathrm{DHG}$ yields but seemingly do not destroy the meso-selectivity.

Inspired by the above observations, we intermediately performed polygridization (in Fig. $2 \mathrm{a}$ ) of $\mathrm{A}_{2} \mathrm{~B}_{2}$-type dicrescentshaped monomers (called DCs). To efficiently obtain the target polymers of Drawing Hands gridarenes (called PDHGs) instead of insoluble cross-linked polymers ( $\mathrm{CPs}$ ), we selected the conditions favoring monosubstitution $\left(\mathrm{CF}_{3} \mathrm{COOH}\right.$ additive, DCM solvent). Unfortunately, the DC-F monomer still afforded the $\mathrm{CP}-\mathrm{F}$ in at least $80 \%$ yields ( $1 \mathrm{~min}$ after the reaction), even if the DC-F concentration $(1 \sim 100 \mathrm{mM})$ was further optimized. These results probably originate from ring-chain competition kinetics between the formation of intermolecular $\mathrm{C}-\mathrm{C}$ bonds and the pathways of intramolecular gridization ${ }^{42}$. However, we observed that CP-F was not generated until $40 \mathrm{~s}$ of reaction. As a result, $15 \sim 25 \mathrm{~s}$ after the initiation, we added $\mathrm{MC1}$ to terminate the active chain ends (followed by quenching in $1 \mathrm{~min}$ ) and fortunately obtained PDHG-F (in $\sim 60 \%$ yields after Soxhlet extraction) without CP-F (Supplementary Fig. 7). Further, even if terminating in $20 \mathrm{~s}$, the slow quenching ( $15 \mathrm{~min}$ after the polygridization) still efficiently generated insoluble CP-F, which is related to thermodynamic equilibrium toward a ring-opening process (Supplementary Fig. 8). Consequently, quick termination and quenching is crucial to the polygridization. For the PDHG-F backbones, the ratio of meso:rac was calculated to be 8.8:1 via the hydrogen nuclear magnetic resonance $\left({ }^{1} \mathrm{H} \mathrm{NMR}\right)$ spectra (see below), corresponding to $79.5 \%$ de of the meso-selectivity (Fig. 2d). Such stereocontrol efficiency, consistent with the gridization time effect of $\mathrm{MC1}(<1 \mathrm{~min})$, is almost maintained when altering reaction times $(6 \sim 25 \mathrm{~s})$ and DC-F concentrations (15 60 mM, in Supplementary Fig. 9). In addition, the other polygridization from the monomer DC-SFX still afforded the meso-selective polygrids PDHG-SFX ( $50 \%$ yields after Soxhlet extraction) but the formation of cross-linked byproduct CP-SFX was unavoidable in at least $\sim 30 \%$ yields, as similar to the effect of the SFX group on the gridization yields.

Characterizations on the backbone tacticity. To demonstrate the structures of DHG products, we grew single crystals via slow evaporation from methanol/DCM mixtures. These crystals reveal that each DHG backbone possesses an inner pore consisting of 18 $s p^{2}$-carbon atoms and $2 s p^{3}$-carbon atoms in a centrosymmetric distribution (Fig. 3a). The two chiral quaternary carbon atoms at the 9-site of DAF moieties serve as the gridarene vertices. The conjugated linkage between the DAF (at the 7-site) and $\mathrm{Cz}$ moieties forms the gridarene edges. Each edge adopts the anticonformation where two 9-sites of the DAF and $\mathrm{Cz}$ moieties are oriented in opposite directions. In detail, meso-DHG1 and racDHG1 are differentiated by their crystal cells and geometric backbones. For meso-DHG1 with the space group P-1, it shows a $\mathrm{C}_{2}$-symmetric backbone with a geometric size of $1.34 \times 0.98 \mathrm{~nm}^{2}$. By contrast, with the space group $\mathrm{C} 12 / \mathrm{c} 1, \mathrm{rac}-\mathrm{DHG} 1$ exhibits an asymmetric folded scaffold with a geometric size of $1.35 \times$ $0.91 \mathrm{~nm}^{2}$. For the two scaffolds, meso-DHG1 has dihedral angles of $129.0^{\circ}$ on the vertices and $43.8^{\circ}$ on the edges, whereas rac-DHG1 exhibits dihedral angles of $108.0^{\circ}$ on the vertices and $14.2^{\circ}$ on the edges.

The backbone differences further discriminate the infrared vibrational transitions between meso- and rac-configurations, which is shown in the Fourier transform infrared spectra (FT-IR) in Supplementary Fig. 10. For the $1024 \mathrm{~cm}^{-1}$ (C-O stretching vibrations) and the $1100 \sim 1080 \mathrm{~cm}^{-1}$ (C-H scissoring vibration on the methoxybenzenes) bands, the folded rac-DHG1 exhibits much stronger intensities than those of $\mathrm{C}_{2}$-symmetric mesoDHG1 whose centrosymmetrically vibrational distribution causes little changes in dipole moment (Supplementary Fig. 11). For asymmetrically vibrational modes, the centrosymmetric backbone of meso-DHG1 enhances infrared vibrational activities both at the $1248 \sim 1264 \mathrm{~cm}^{-1}$ (asymmetric C-O stretching vibrations) and the $1294 \mathrm{~cm}^{-1}$ (asymmetric C-H scissoring vibration on the DAF groups) bands vs. the asymmetric configuration of rac-DHG1. More interestingly, the band intensity ratio of $1294 \mathrm{~cm}^{-1}$ to $1024 \mathrm{~cm}^{-1}\left(I_{1294} / I_{1024}\right)$ is gradually enhanced from 0.13 to 0.76 when increasing the meso-DHG1 proportion from 0 to $100 \%$ in the mixing systems (Fig. 3b and Supplementary Fig. 12). Meanwhile, the band intensity ratio of $1248 \sim 1264 \mathrm{~cm}^{-1}$ to $1024 \mathrm{~cm}^{-1}$ $\left(I_{1248} / I_{1024}\right)$ is continuously increased from 0.56 to 1.55 . The above vibrational features of meso-DHG1 are also observed in PDHG-F that exhibits $I_{1294} / I_{1024}=0.66$ and $I_{1248} / I_{1024}=1.40$, which probably reveals $80 \%$ de of meso-configurations.

The structural differences between DHG diastereomers were further demonstrated by the shielding/deshielding effect of aromatic ring currents in ${ }^{1} \mathrm{H}$ NMR spectra (Fig. $3 \mathrm{c}$ and Supplementary Figs. 13-18). For meso-DHG1 and meso-DHG4, the ring currents of DAF moieties exclusively shield the $a$ and $b$ protons on the methoxybenzyl groups, which is evidenced by their chemical shifts $(6.95 \sim 6.65$ parts per million (p.p.m.)) being smaller than 
$\mathbf{a}$

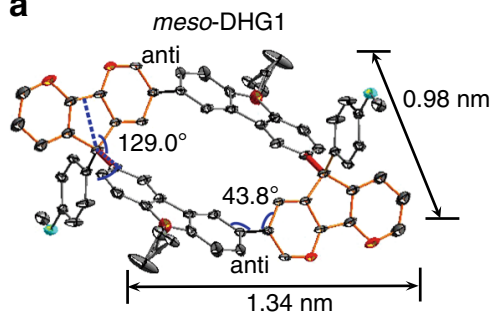

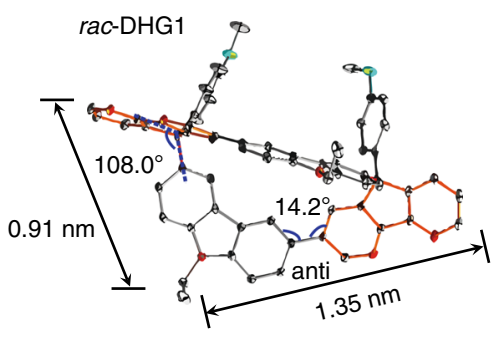

b

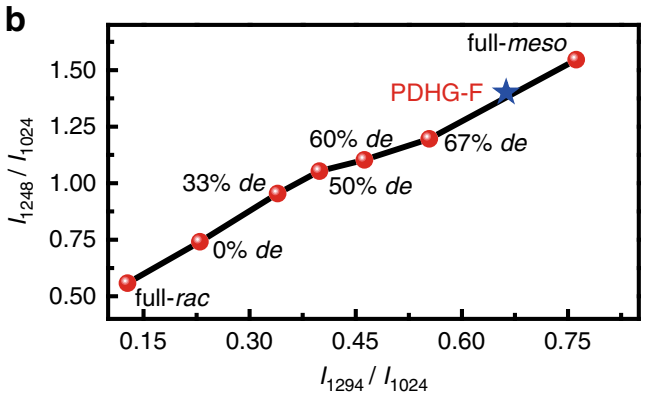

C
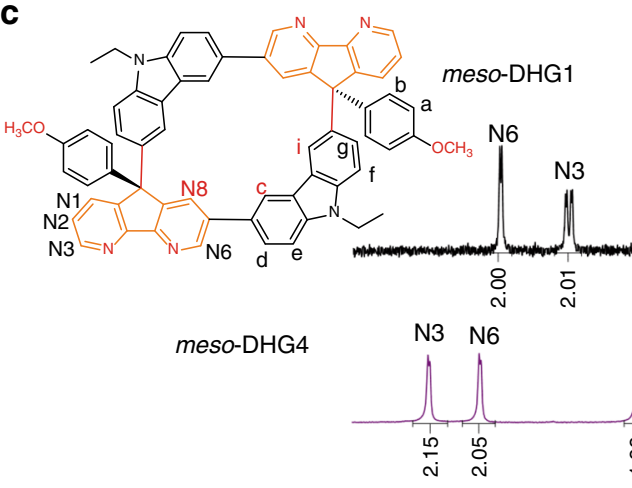

PDHG-F

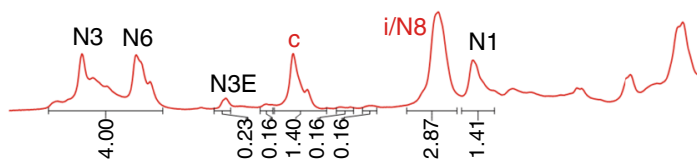

rac-DHG4

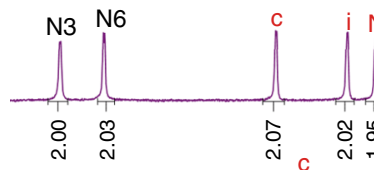

rac-DHG1

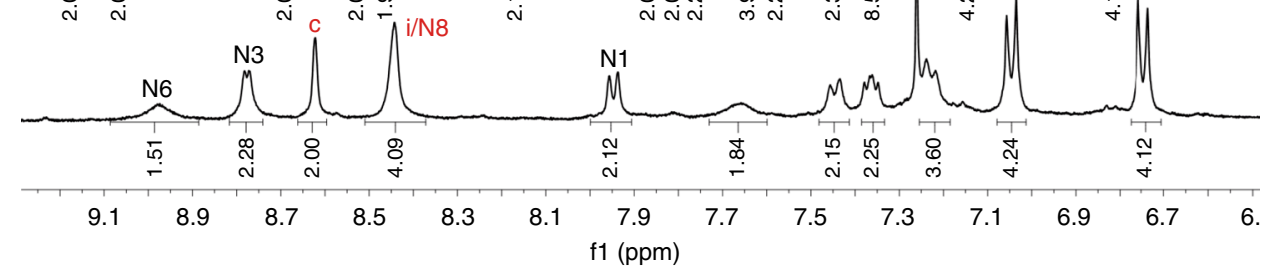

Fig. 3 Characterizations of the tacticity of DHGs and PDHG-F backbones. a The single-crystal crystallography of meso-DHG1 and rac-DHG1s. The blue lines exhibit the dihedral angles of the backbones. $\mathbf{b}$ The integral ratio of the vibrational absorption at $1248 \sim 1264 \mathrm{~cm}^{-1}$ band to $1024 \mathrm{~cm}^{-1}$ band $\left(I_{1248} /\right.$ $\left.I_{1024}\right)$ and the intensity ratio of the $1294 \mathrm{~cm}^{-1}$ band to $1024 \mathrm{~cm}^{-1}$ band $\left(I_{1294} / I_{1024}\right)$ for the specific mixing systems (meso-DHG1 and rac-DHG1). These results are extracted from the Fourier transform infrared spectra of mixing samples with well-defined doping percentage (transformed to de value of mesoselectivity). For example, $0 \%$ de $=1: 1$ of meso-DHG1: rac-DHG1; full-rac represents the pure rac-DHG1 sample; full-meso represents the pure mesoDHG1 sample. The red dots exhibit the band intensity ratio of DHG1 mixing systems; the blue star shows the band intensity ratio of PDHG-F. $\mathbf{c}$ The ${ }^{1} \mathrm{H}$ NMR spectra of DHG1 (in black lines, as the end-capping units), DHG4 (in purple lines, as the repeat units), and PDHG-F (in red lines). The assignments of mesoDHG4 were made in reference to the ${ }^{1} \mathrm{H}^{1} \mathrm{H}$ correlation spectroscopy results. The key positions associated with the stereoselective calculation (such as $\mathrm{c}$, $\mathrm{i}$, and N8 sites) are marked in red.

those in rac-DHG1 and rac-DHG4 (7.15 6.75 p.p.m.). For both rac-DHGs, the folded aromatic scaffold enhances the deshielding effect on the protons of the inner pores, displaying a chemical shift in the $i$ and $\mathrm{N} 8$ protons $(8.55 \sim 8.40$ p.p.m.) larger than that in both meso-DHGs $(8.40 \sim 8.30$ p.p.m.). The above observations will be applied in PDHG-F if their chain conformations are expanded rather than collapsed, because chain collapse induces compact intramolecular stacking, which upshifts overall proton signals ${ }^{6}$. According to the results of the molecular dynamic simulation, we evaluated the expansion factor $\beta>1$ for PDHG-F chains in $\mathrm{CHCl}_{3}$ solvent (detailed in Supplementary Note 4), indicating the expanded conformations. Such evaluation is confirmed by the overall proton signals of PDHG-F with approximately the same chemical shifts as DHG4. Thus, the $c$, i/N8 protons, with discriminated chemical shifts between the meso- and rac-configurational repeat units, can be used to calculate the de value of meso-selectivity via the integral ratio. For the $c$ sites of PDHG-F, the integral ratio in 8.65 p.p.m. (consistent with meso-DHG4, an integration of 1.40) to 8.70 p.p.m. (identical to rac-DHG4, an integration of 0.16 ) displays the $79.5 \%$ de of the meso-selectivity. For the $i / \mathrm{N} 8$ sites of PDHG-F, the total integral ratio in 8.32 p.p.m. (consistent with meso-DHG4, the total integrations of 2.87 ) to $8.54 \sim 8.48$ p.p.m. (identical to rac-DHG4, the total integrations of 0.32 ) evaluates the $79.9 \%$ de of mesoconfiguration. Moreover, these meso-selective results of PDHG-F are reconfirmed by their chemical shift distribution of $a, b$ signals that are dominantly assigned to meso-DHGs, according to the ${ }^{1} \mathrm{H}-{ }^{1} \mathrm{H}$ correlation spectroscopy (Supplementary Fig. 18).

Characterizations on the linear PDHG-F backbone. Given the absence of a branched scaffold, we hypothesized that PDHG-F would possess rod-like chain properties. In this regard, its 
Mark-Houwink exponent $\alpha$ would be $\sim 1.7$ instead of $0.3 \sim 0.5$ (for branched backbones ${ }^{43}$ ) ${ }^{44}$. The Mark-Houwink exponent of PDHG-F $\left(\alpha_{\mathrm{LP}}\right)$ can be deduced by its gel permeation chromatography (GPC) calibration curve that shows a linear relationship between logarithmic molecular mass $(\lg M)$ and elution time $(t)^{9}$. Its curve slope $\left(k_{\mathrm{LP}}\right)$ can transform into $\alpha_{\mathrm{LP}}$ via the equation (detailed in Supplementary Note 5): $\left(\alpha_{\mathrm{LP}}+1\right) /\left(\alpha_{\mathrm{PS}}+1\right)=k_{\mathrm{PS}} /$ $k_{\mathrm{LP}}$, based on the known values including the slope of the polystyrene (PS) calibration curve $\left(k_{\mathrm{PS}}=-0.198\right.$, during the elution time of $18 \sim 23 \mathrm{~min}$ ) and the Mark-Houwink exponent of PS $\left(\alpha_{\mathrm{PS}}=0.714\right)^{45}$. To obtain $k_{\mathrm{LP}}$, we analysed the chromatograph of PDHG-F with an average lower molecular weight and tentatively assigned their elution peaks $(19 \sim 23.4 \mathrm{~min}$, all in $\sim 1.01$ polydispersity index) to corresponding degree of polymerization (DP) of $2 \sim 6$ (Fig. $4 \mathrm{a}$ ). In this case, we established a calibration equation $\lg M=-0.128 t+6.329$ where $k_{\mathrm{LP}}=-0.128$ transforms into $\alpha_{\mathrm{LP}}=1.651$. Therefore, such high $\alpha_{\mathrm{LP}}$ reconfirms our hypothesis of the rod-like main chain for PDHG-F backbones (up to DP =6).

Deeply, we demonstrated the PDHG-F scaffolds with higher DP via calculating the number of chain ends $\left(N_{\text {end }}\right)$. Theoretically, the linear scaffold displays $N_{\text {end }}=2$, whereas the branched scaffold shows $N_{\text {end }} \geq 3$. $N_{\text {end }}$ can be evaluated ${ }^{6}$ through the equation $N_{\text {end }}=\left(\mathrm{DP} \times 4 I_{\mathrm{N} 3 \mathrm{E}}\right) /\left(I_{\mathrm{N} 3+\mathrm{N} 6}+I_{\mathrm{N} 3 \mathrm{E}}\right)$, where $I_{\mathrm{N} 3 \mathrm{E}}$ is the integration of the chain-end $\mathrm{N} 3 \mathrm{E}$ proton signals at 8.80 p.p.m. and $I_{\mathrm{N} 3+\mathrm{N} 6}$ is the total integrations of the $\mathrm{N} 6$ and $\mathrm{N} 3$ protons at $9.15 \sim 8.95$ p.p.m. (in Fig. $3 c$ ). To obtain the average DP, we divided the elution peak $(15 \sim 22.5 \mathrm{~min})$ in the light of specific elution times corresponding to individually well-defined DP (secondary elution peaks in Fig. 4b), based on the extrapolated calibration curve of the PDHG-F system (Supplementary Table 3). Such division can obtain the fraction of each specific DP, which transforms into the height of red stars in Fig. 4b. Further, via discretely integrating each DP value in individual fraction (according to equations ES12 and ES13 in Supplementary Note 5), we calculated the number- and weight-average degrees of polymerization $\mathrm{DP}_{n}=8.5$ and $\mathrm{DP}_{\mathrm{w}}=9.5$, respectively. $\mathrm{DP}=8.5$ transforms into $N_{\text {end }}=1.85$, which indicates the linear chains with a maximum DP of 12 ( $\sim 30 \mathrm{~nm}$ in length, $35.4 \%$ proportion).

Moreover, a molecular weight dependence of hydrodynamic radius $\left(R_{\mathrm{h}}\right)$ was tested to demonstrate the rigidity of PDHG-F ( $R_{\mathrm{h}} \sim M^{1}$ for stretched chains) via dynamic light scattering (DLS). According to the size distribution (Supplementary Fig. 21), we obtained their $R_{\mathrm{h}}=11.1 \sim 14.2 \mathrm{~nm} \quad\left(\mathrm{DP}_{\mathrm{w}}=9.1 \sim 10.2\right)$, which represents the radius of the hydrodynamic sphere model (Fig. 4c). As these sizes are slightly longer than the half of a PDHG-F main chain, we deduced that this spherical diameter is similar to a PDHG-F backbone. Further, the scaling law of

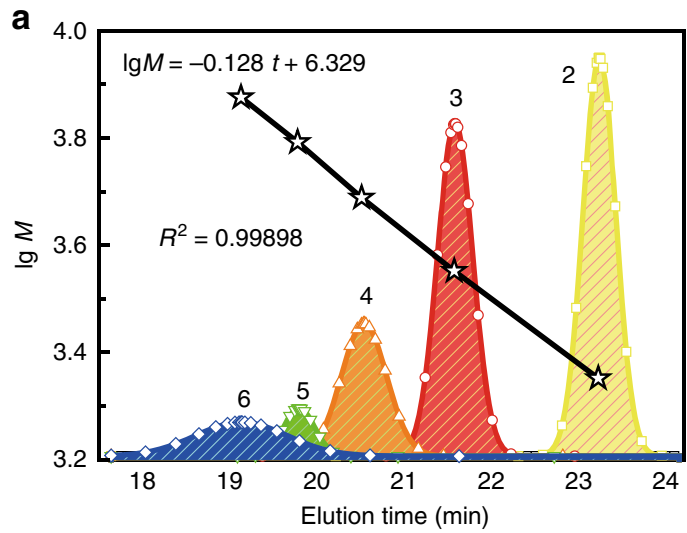

b
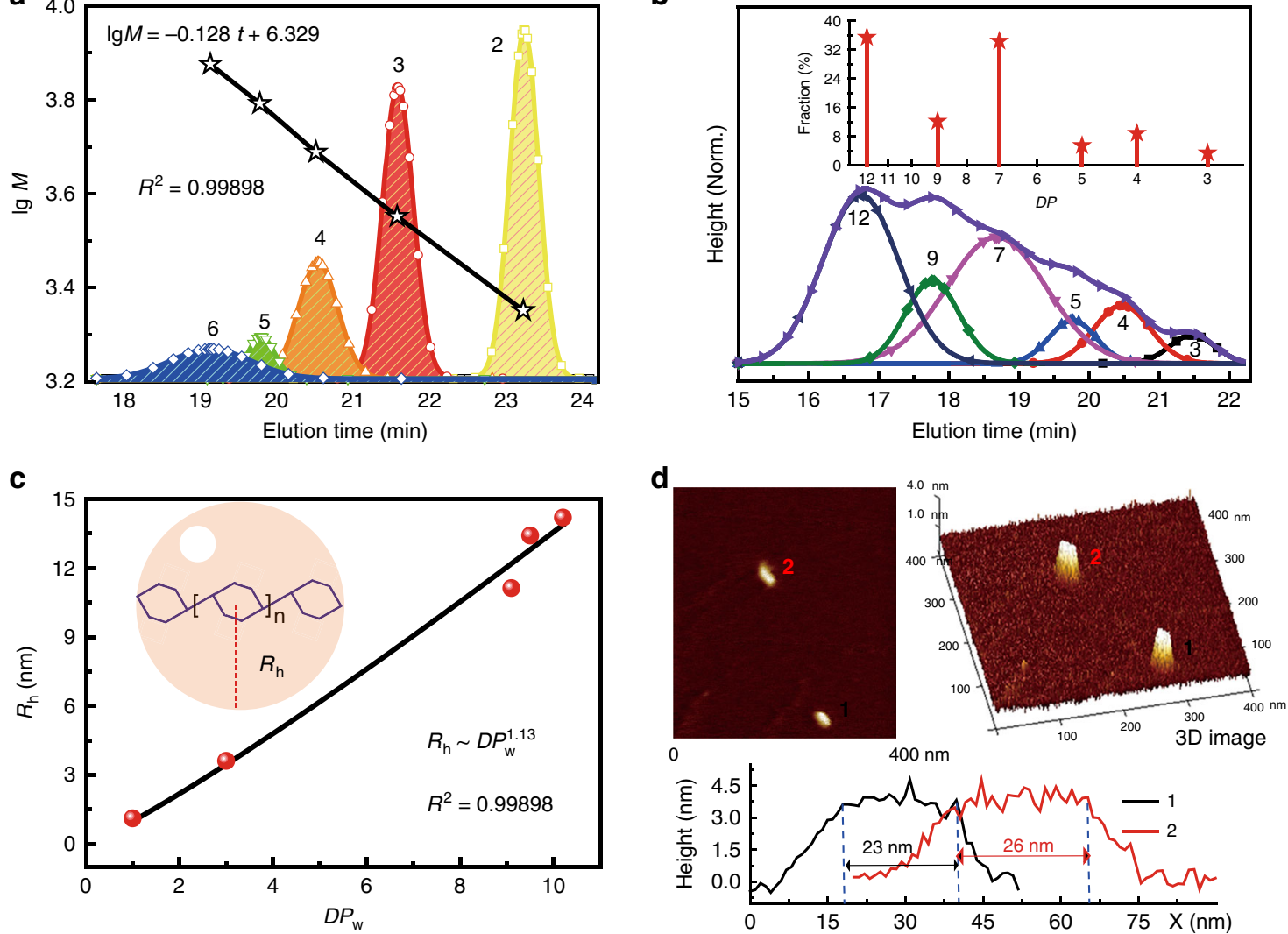

Fig. 4 Characterizations of the linear configuration of PDHG-F main chains. a The calibration of PDHG-F with lower molecular weight (the coefficient of determination $R^{2}$ is 0.99898 ). The yellow, red, orange, green, and blue peaks corresponds to dimer, trimer, tetramer, pentamer, and hexamer, respectively (in which the star dots exhibit their individual logarithmic molecular mass $\lg M$ and elution time $t$ ). $\mathbf{b}$ The GPC analysis of PDHG-F. The broad elution peak (violet lines) is divided into a series of gauss peaks (in black, red, blue, magenta, olive, and navy lines) with well-defined degree of polymerization, in terms of the extrapolation of PDHG-F calibration. The height of red star columns represents the proportion of each degree of polymerization. $\mathbf{c}$ The hydrodynamic radius $\left(R_{\mathrm{h}}\right)$ of PDHG-F with different weight-average degrees of polymerization (DPs), marked in red dots. The black lines exhibit the DP dependence of hydrodynamic radius $R_{\mathrm{h}}\left(R^{2}=0.98473\right)$. The blue lines depict the linear PDHG-F backbones. Its hydrodynamic model in solutions is represented as the orange sphere whose radius (red line) is identical to $R_{\mathrm{h}}$. $\mathbf{d}$ The atomic force microscope images of the PDHG-F chains (two chains labeling 1 and 2 ) including 2D and 3D image. In the height analysis, the size between two blue dashed lines (above $3.2 \mathrm{~nm}$ ) exhibits the chain length of visualized PDHG-F (black for 1 and red for 2). 
$R_{\mathrm{h}} \sim \mathrm{DP}_{\mathrm{w}}^{1.13} \sim M^{1.13}$ approximately exhibits the linear chain conformation with high rigidity (up to $\sim 28 \mathrm{~nm}$ ). Such rod-like backbones are further visualized by atomic force microscopy. In Fig. 4d, two PDHG-F chains individually display $23 \mathrm{~nm}$ (labeling 1) and $26 \mathrm{~nm}$ (labeling 2) in length, which are approximate to the DLS results.

Gridization mechanism. Based on the above experimental results, we performed mechanistic studies on the gridization process between two MC1 substrates. In particular, via nuclear Overhauser effect spectroscopy (NOESY, in Supplementary Fig. 22a), the protonated MC1 substrates were analysed to adopt both anti- and syn-conformations in solution. Further, the formation of DHG1 results from three conformational combinations (Fig. 5a) as follows: (i) two syn-conformations (I, II); (ii) mixed anti- and syn-conformations (III, IV); and (iii) two anti-conformations (V, VI). Among of them, overall rac-configurations (II, IV, and VI for 12.0, 12.4, and $0 \mathrm{kcal} \mathrm{mol}^{-1}$, respectively) are more stable than meso-configurations (I, III, and V for 90.3, 18.9, and $4.9 \mathrm{kcal} \mathrm{mol}^{-1}$, respectively). In this regard, the thermodynamic pathway is not the rate-determining step during the meso-selective gridization.

Furthermore, considering the aggregation effect on the gridization pathways, we deduced that the formation of DHG1 is possibly related to the process of dimer packing between two MC1 molecules. Given the donor-acceptor packing mode within multicationic repulsion ${ }^{39}$, we hypothesized that two MC1 superelectrophiles would adopt an antiparallel stacking geometry in face-to-face orientation between a DAF group and a
$\mathrm{Cz}$ group. Such hypothesis is confirmed by the space correlations in the NOESY spectrum (Supplementary Fig. 22b). Moreover, the PDHG-F products generated from polygridizations also reconfirm the antiparallel stacking process. According to the orientation of two methoxybenzyl groups (Fig. 5b), the antiparallel stacking can be categorized into the centrosymmetric geometry VII (in the opposite orientations that generate meso-DHG1) and the asymmetric geometry VIII (in the same orientation that obtains rac-DHG1). In detail, the centrosymmetric stacking orients two DAF groups in the opposite directions and thus suffers from less intermolecular coulombic repulsion ${ }^{39}$ (especially at the 4,5-sites of DAF groups) than the asymmetric stacking, which is similar to other pyridinium derivatives ${ }^{29,30}$. More significantly, the intramolecularly tricationic repulsion induces charge delocalization ${ }^{46}$ that transforms the methoxybenzyl moiety into the planarized quinonemethide-type structure ${ }^{38}$, which further strengthens the antiparallel and centrosymmetric packing for meso-selectivity (Supplementary Fig. 23). Such transformation is confirmed by the rac-selectivity from tolylbased MC2 substrates in the absence of quinonemethide-type structures. Regarding the meso-stereocontrol results, we deduced that the more stably antiparallel stacking could dominate the reaction pathways that merely generate I, III, and V conformations. However, the single-crystal crystallography of meso-DHG1 and rac-DHG1 demonstrate the formation of $\mathrm{V}$ (major products) and VI (minor products), whereas both I and III with much higher conformational energies were not observed. In all, the powerfully centrosymmetric packing results in the meso-selectivity, under the prerequisite conditions of relatively stable products' conformations.
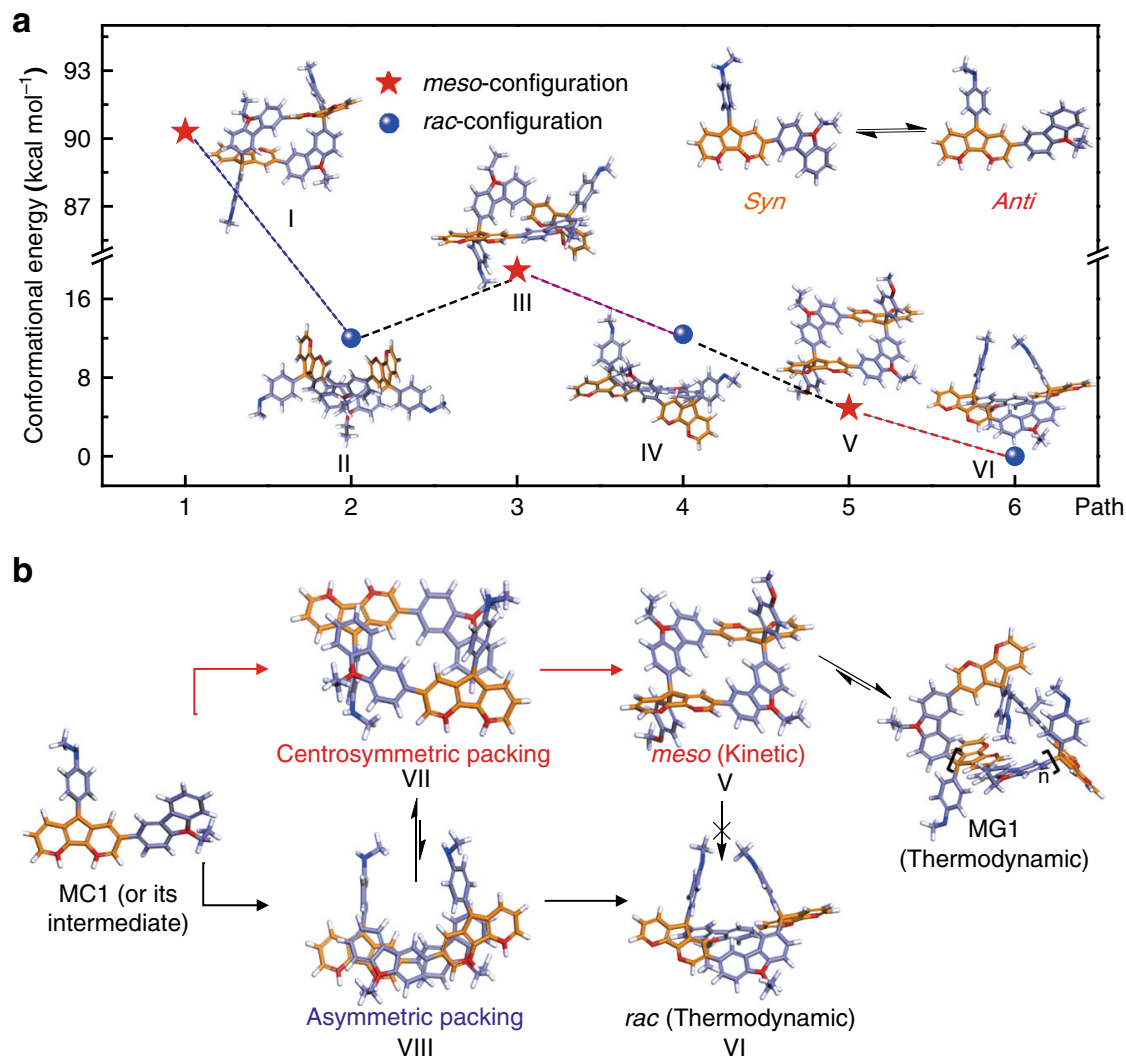

Fig. 5 The plausible gridization mechanisms. a The relative energies of the DHG1 products with different conformations (from individual reaction pathways). The relative energy of anti-anti-conformational rac-DHG1 is defined as $0 \mathrm{kcal} \mathrm{mol}^{-1}$. Both I and II consist of two syn-conformational MC1 substrates; both III and IV consist of an anti-conformational MC1 and a syn-conformational MC1; both V and VI are composed of two anticonformational $\mathrm{MC} 1$ substrates. The red stars and blue beads represent the meso-configurations and the rac-backbones, respectively. $\mathbf{b}$ The gridization mechanism of MC1. 
Taken together, the above results reveal that the real gridization pathway should be determined by the competition between the kinetic stacking stage and the thermodynamic bonding stage. The strong noncovalent interactions enhanced by quinonemethidetype structures form the stable antiparallel and centrosymmetric assembly VII, which overcomes the thermodynamic barrier to dominantly generate meso-DHG1. Further, meso-DHG1 transforms into MG1 during which the thermodynamic control is favorable to the ring-opening process toward the formation of MG1 (Fig. 5b). On the contrary, due to the absence of strong self-assembly (confirmed by NOESY that does not display corresponding cross peaks, in Supplementary Fig. 24) and quinonemethide-type backbones, the methyl-based MC2 substrates are subjected to the thermodynamic control that facilitates the rac-selectivity and random oligomerization pathways.

The DC-F monomers are similarly controlled by the above packing mode, which not only maintains the meso-selectivity but also results in the abnormal polymerization kinetics (contradictory to the second-order rate rules ${ }^{42}$, shown in Supplementary Figs. 25 and 26). According to primary results, enhancing the DC-F concentration from 15 to $60 \mathrm{mM}$ leads to decreasing the $\mathrm{DP}_{n}$ from 9.2 to 8.1 rather than increasing in proportional manners; prolonging the polygridization time (before termination) from 6 to $25 \mathrm{~s}$ slightly increases the $\mathrm{DP}_{n}$ from 8.4 to 9.2, without proportional modes as well. These results indicate that the supramolecular packing enables to consistently change reaction rate constant in situ.

Simulations of chain collapse in different tacticity. Inspired by the analogous tacticity in polyesters ${ }^{37}$ and poly(vinylether) $\mathrm{s}^{22}$, we study the effect of meso-configurations on the chain collapse via atomistic simulation. As the enthalpic driving force ${ }^{47}$ is crucial to the collapse of stiff backbones ${ }^{48}$, both PDHG-F chains (full mesoconfigurational meso-PDHG-F and full rac-configurational racPDHG-F individually) are simulated in vacuum that provides dominantly intramolecular segment-segment interactions ${ }^{49}$. During the dynamic equilibrium (Constant volume/constant temperature ensemble (NVT), $298 \mathrm{~K}$ ), meso-PDHG-F performs the conformational transition from the linear rod (expanded chains (EC stage)), via the folded sausage (sausage-like chains (SC stage)) to the collapsed rod (cluster stage, in Fig. 6a). The eccentricity of its ellipsoid model is relatively decreased from 0.99 to 0.91 (Fig. 6b), which still exhibits highly structural anisotropy that is similar to the main chains of one-dimensional nanopolymers ${ }^{50}$. In contrast, rac-PDHG-F undergoes the conformational transition from the helical backbone (EC stage), via the cyclic skeleton (SC stage) to the toroid main chain (cluster stage). The eccentricity of its ellipsoid model is drastically fluctuated $(0.96 \sim 0.11)$ and is equilibrated at $0.47 \sim 0.57$, which is obviously lower than that of meso-PDHG-F (shown in Fig. 6b). Both collapsed main chains, distinguished from traditional globule manners ${ }^{51}$, were predicted merely with coarse-grained models ${ }^{48}$. During the collapsed process, meso-PDHG-F undergoes the rotation of $\mathrm{Cz}$ groups toward syn-type $\mathrm{DHG}$ edges to crumple the chains, whereas the rotation between $\mathrm{C}_{2}$-symmetric meso-DHG units and fluorenyl groups maintains the linear backbones. Conversely, the chain collapse of rac-PDHG-F originates from the rotation between the folded rac-DHG units and fluorenyl groups. The above distinguished conformational transformations result in various covalent energies (valence in Fig. 6c) that are linked to the structural stability of collapsed conformations. For example, due to structural instability stemming from syn-type DHG edges, the collapsed meso-PDHG-F possesses higher average covalent energy $\left(7.3 \mathrm{kcal} \mathrm{mol}^{-1}\right.$ per repeat units) than that of collapsed rac-PDHG-F (Fig. 6c). This result also suggests that the meso-configured repeat units enhance the ability to prevent chain collapse, which indirectly increased the backbone rigidity vs. rac-configuration. Further, these features are majorly maintained in other chain length (DP $=7,9$, and 18, Supplementary Figs. 27-29), ruling out the entropic effect on such chain relaxation ${ }^{49}$. In terms of above collapsed behaviors, PDHG-F chains in full meso-configuration are more likely to serve as the rigid nanopolymer models.

\section{Discussion}

We demonstrated the gridization rule of $\mathrm{A}_{n} \mathrm{~B}_{m}$-type synthons under different internal and external conditions, and discovered the stereoselective gridization that is kinetically controlled rather than thermodynamically controlled processes during ultrafast superelectrophilic Friedel-Crafts reaction. As a result, mesoconfigurational Drawing Hand-type gridarenes are efficiently synthesized and confirmed by various tools such as singlecrystalline X-ray diffraction, NMR, FT-IR, and so on. The stereodetermining step stems from the centrosymmetric packing of superelectrophilic species via synergistically $\pi-\pi$ stacking attractions and intermolecular multicationic repulsions. We further examine the gridization rule of $\mathrm{A}_{2} \mathrm{~B}_{2}$-type monomers into polygrids that are an alternative to synthetic methodology of ringchain-alternating polygridarene backbones. As a result, such meso-stereocontrol is genetically transformed into polygridization under the initial stage that support the hypothesis of antiparallel molecular packing modes. To understand tacticity of polygrids, molecular dynamic simulations are made to visualize the differences of chain collapse between meso- and rac-polygrids. Compared with rac-polygrids, meso-polygrids exhibit rod-like conformations and increased ability against chain collapse, which reveals higher backbone rigidity. Moreover, these stereoregular nanopolymers exhibit microscopic dynamics distinctive from traditional polymers, which will fundamentally impact on the polymer science. Our gridization and polygridization afford powerful molecular installing nanotechnology for the innovation of covalent nano-linkages with the multi-bonding feature and the multiscale molecules with the potential application of organic electronic materials, devices, and intelligence.

\section{Methods}

Materials. The synthetic procedures and details of the Friedel-Crafts reaction, gridization, and polygridizations are described in the Supplementary Information files. The preparation of substrates are detailed in the Supplementary Information files as well.

Structural characteristics via NMR technologies. ${ }^{1} \mathrm{H}$ and ${ }^{13} \mathrm{C}$ NMR spectra were acquired from a Bruker $400 \mathrm{MHz}$ NMR Fourier transform spectrometer ( $400 \mathrm{MHz}$ and $100 \mathrm{MHz}$, respectively) at $20^{\circ} \mathrm{C}$. The meso-DHG4, rac-DHG4, and PDHG-F samples were characterized by the ${ }^{1} \mathrm{H}$ NMR Fourier transform spectrometer in 600 $\mathrm{MHz}$. Chemical shifts are shown as $\delta$ in units of p.p.m. relative to an internal standard $\left[{ }^{1} \mathrm{H}\right.$ NMR: tetramethylsilane $=0.00$ p.p.m. $]$ or relative residual peaks ( ${ }^{1} \mathrm{H}$ NMR: 7.26 for $\mathrm{CDCl}_{3}, 2.50$ for $d_{6}$-DMSO; ${ }^{13} \mathrm{C}$ NMR: 77.0 triplet for $\mathrm{CDCl}_{3}$ 39.25 for $d_{6}$-DMSO). In brief, multiplicities of every signal peak are shown as follows: s (singlet); d (doublet); t (triplet); q (quartet); dd (doublet of doublets); dt (doublet of triplets); $\mathrm{m}$ (multiplet). Coupling constants are expressed as a $J$-value in $\mathrm{Hz}$. To calculate the meso-selectivity during the gridization, the reaction solution was quenched with $\mathrm{KOH}$ aqueous solution and then was extracted with $\mathrm{CH}_{2} \mathrm{Cl}_{2}$ solvents. Then, we heated the organic phase to remove the $\mathrm{CH}_{2} \mathrm{Cl}_{2}$ solvent and added $\mathrm{CDCl}_{3}$ solvent to perform the NMR measurements. ${ }^{1} \mathrm{H}-{ }^{1} \mathrm{H}$ correlation spectroscopy $\left({ }^{1} \mathrm{H}-{ }^{1} \mathrm{H}\right.$ COSY $)$ was performed to assign the protons in meso-DHG4, protonated meso-DHG1, and PDHG-F. NOESY was performed to demonstrate the likely stacking mode of protonated $\mathrm{MC1}$ and $\mathrm{MC}$.

Measurements of molar mass with mass spectrometry. Matrix-assisted laser desorption/ionization time-of-flight mass spectrometry was utilized to obtain the molecular mass of substrates. The DHG products with $900 \sim 1100 \mathrm{~m} / \mathrm{z}$ and small molecules $(<750 \mathrm{~m} / \mathrm{z})$ were characterized by High Resolution Mass Spectrometry, in brief, HRMS (Thermo Fisher Scientific, Linear ion Trap Fourier Transform ion Cyclotron Resonance Mass Spectrometry, in brief, LTQ-FTICR-MS). 
a

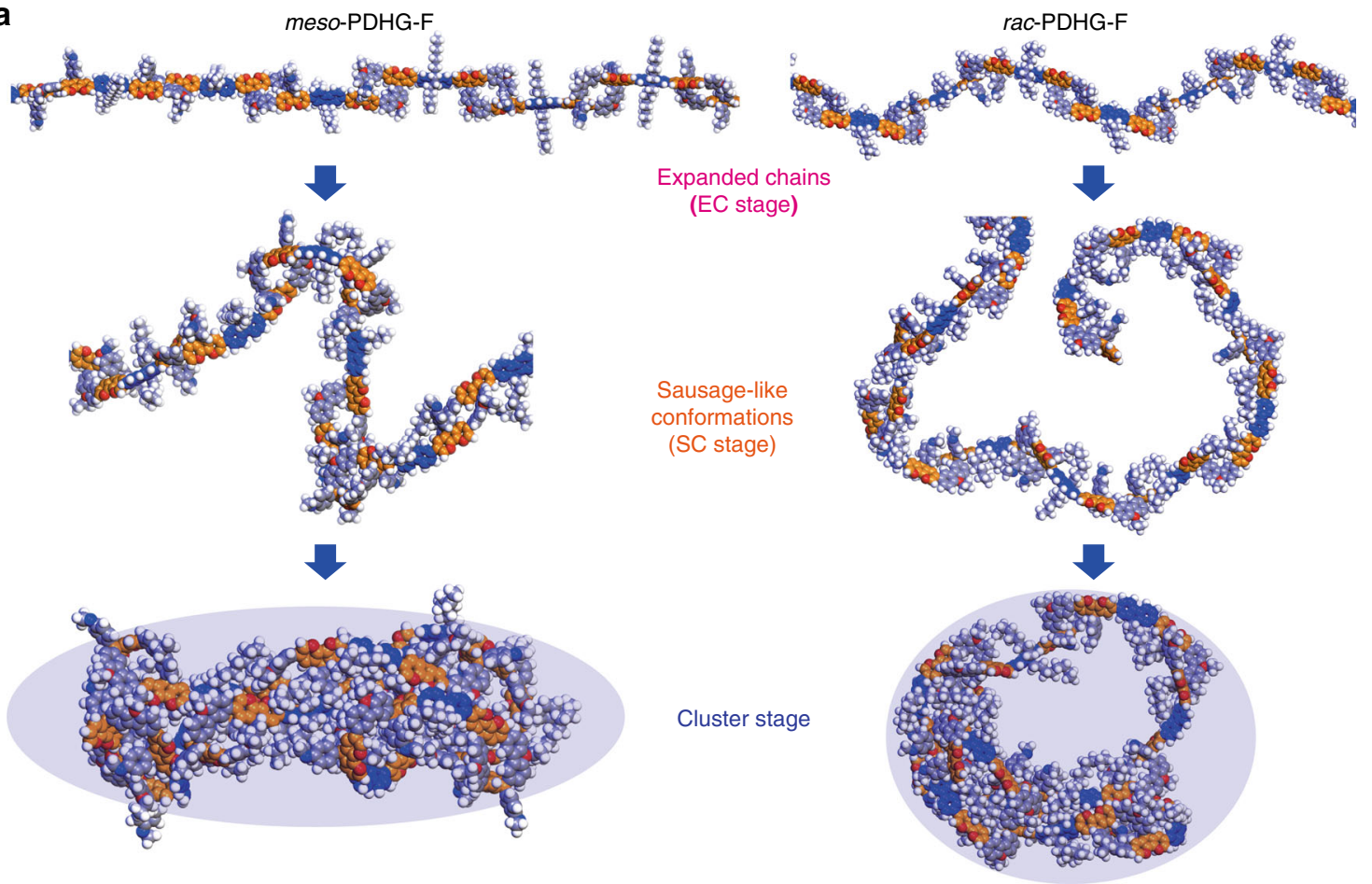

b
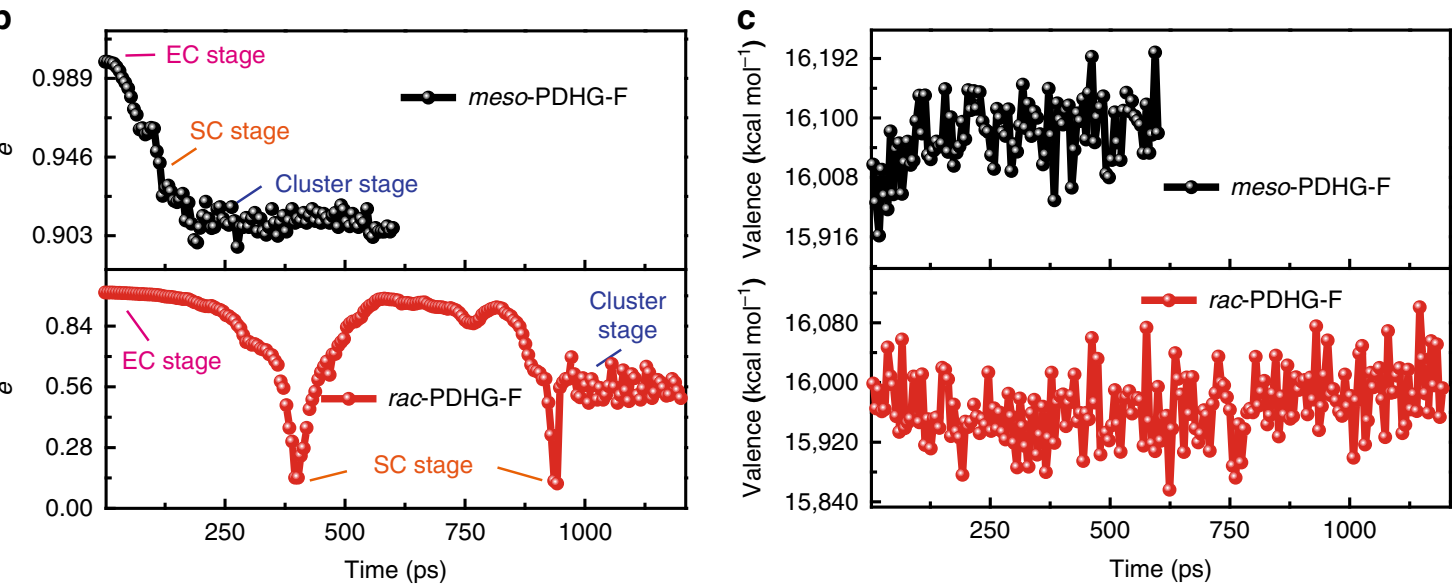

Fig. 6 The molecular dynamic simulation of the single PDHG-F chain collapse under the vacuum conditions (298 K). Meso-PDHG-F is defined as the main chain with $100 \%$ de of meso-configuration; rac-PDHG-F polygrids is represented as the backbone exhibiting $100 \%$ de of rac-configuration. a The conformations ( $D P=13$ ) of expanded chains (EC stage), sausage-like chains ( $S C$ stage), and collapsed chains (CC stage) during the collapsed process. The blue region displays the ellipsoid model of PDHG-F backbones that completely covers the main chain. $\mathbf{b}$ The eccentricity (e) of the ellipsoid model (blue region) for quantitatively evaluating the conformational anisotropy ( $e=1$ for the $100 \%$ anisotropy and $e=0$ for the perfect isotropy) of meso-PDHG$\mathrm{F}$ (black) and rac-PDHG-F chains (red). c The covalent energy (valence) of the main chain during the collapse of the meso-PDHG-F (black) and rac-PDHG-F (red) backbones.

Single-crystal crystallography. X-ray diffraction was performed on a Bruker D8 $\mathrm{X}$-ray diffractometer with $\mathrm{Cu}$ Ka radiation $(\lambda=1.54050 \AA)$. The operating $2 \theta$ angle was in the range of $5 \sim 30^{\circ}$ with a step length of $0.02^{\circ}$. The single-crystal crystallography data were all collected at $\sim 100 \mathrm{~K}$ on a Bruker $2000 \mathrm{CCD}$ area detector with graphite-monochromated Mo Ka radiation $(\lambda=0.71073 \AA)$. To solve the structures of meso-DHG1, meso-DHG4, rac-DHG1, rac-DHG2, and 2a, we utilized direct methods in SHELXS-2015 and refined against F2 via SHELXL-2015. Hydrogen atoms were fixed geometrically and refined isotropically.

Characteristics via FT-IR method. The DHGs and PDHG-F products were addressed as $\mathrm{KBr}$ pellets. For the mixing systems ( $d e=0 \sim 67 \%)$, each system was obtained by mixing meso-DHG1 with rac-DHG1 sample in 1:1, 2:1, 3:1, 4:1, and 5:1 molar ratio. Then the infrared absorptions of these samples were measured by a Fourier transform infrared spectrophotometer (SHIMADZU, IRPrestige-21).
GPC measurements. GPC experiments were conducted on an HP1100 HPLC system possessing 7911GP-502 and GPC columns using PSs as the standards and tetrahydrofuran (THF) as the eluent at a flow rate of $1.0 \mathrm{ml} \mathrm{min}^{-1}$ at $25^{\circ} \mathrm{C}$. The corresponding principles are detailed in the Supplementary Information.

DLS experiments. The DLS characteristics were determined by a Brookhaven instrument (BI-200SM). The concentration of PDHG-F samples were lower than $0.05 \mathrm{mg} \mathrm{ml}^{-1}$ because of the existence of aggregation that drastically amplifies the results of hydrodynamic radius.

Visualization via atomic force microscopy. The atomic force microscopy characteristics were performed under the peak-force tapping mode. In order to reduce the tip-broadening effect, we selected the supersharp tips (type: SAA-HPI-SS). The PDHG-F film on the mica sheet was via spin-coating the solutions (concentration: $3 \mu \mathrm{g} \mathrm{ml}^{-1}$; solvent: 1,2-dichloroethane). 
Single energy of DHG1 conformations. We use the software Material Studio 2016 to calculate single energies of DHG1 conformations I IV (in Fig. 5a) via the DMol3 calculation module. The theoretical calculation is based on the method of B3LYP. The transition state (TS) method was used for the density functional theory with van der Waals dispersion corrections (DFT-D correction).

Atomistic simulation of chain collapse. The software Material Studio 2016 was utilized to simulate the chain collapse. Before performing the dynamics, all PDHG$\mathrm{F}$ chains were constructed in the expanded conformations where the dihedral angle between adjacent repeating units are approximate to $180^{\circ}$. The dynamics of chain collapse were carried out under the conditions of NVT ensemble (298 K) and the COMPASS forcefield (the summation method of van de Waals term and the electrostatic term are both Group-based in which the cutoff distance were set as $12.5 \AA$ ). The total times of dynamics were set as $600 \mathrm{ps}$ for meso-PDHG-F (DP = 13) with the time step of $1.5 \mathrm{ps}$. In fact, the meso-PDHG-F falls into collapsed equilibrium (cluster stage) after 100-250 ps. By contrast, due to much slow relaxation process toward collapsed equilibrium, the total times of dynamics were set as $3000 \mathrm{ps}$ for $\mathrm{rac}$-PDHG-F $(\mathrm{DP}=13)$ with the time step of $1.5 \mathrm{ps}$.

\section{Data availability}

The authors declare that all data supporting the current findings of this study are available in the main manuscript or in the Supplementary Information. The single-crystal datum (including a.cif file and a structural figure) of 2a, meso-DHG1, rac-DHG1, racDHG2, and meso-DHG4 were upload in Cambridge Structural Database, obtaining corresponding CCDC number 1886054, 1886046, 1886053, 1886052, and 1938823, respectively. The source data underlying Figs. 5a and 6a are provided as a Source Data file. Other data are available from the corresponding author on reasonable request.

Received: 24 July 2019; Accepted: 3 March 2020;

Published online: 09 April 2020

\section{References}

1. Markowitz, M. A., Janout, V., Castner, D. G. \& Regen, S. L. Perforated monolayers: design and synthesis of porous and cohesive monolayers from mercurated calix [n] arenes. J. Am. Chem. Soc. 111, 8192-8200 (1989).

2. Ogoshi, T., Kanai, S., Fujinami, S., Yamagishi, T.-a \& Nakamoto, Y. paraBridged symmetrical pillar [5] arenes: their Lewis acid catalyzed synthesis and host-guest property. J. Am. Chem. Soc. 130, 5022-5023 (2008).

3. Tozawa, T. et al. Porous organic cages. Nat. Mater. 8, 973-978 (2009).

4. Chun, Y. et al. Calix[n]imidazolium as a new class of positively charged homocalix compounds. Nat. Commun. 4, 1797-1804 (2013).

5. Brown, C. J. \& Farthing, A. C. Preparation and structure of Di-p-xylylene. Nature 164, 915-916 (1949).

6. $\mathrm{Wu}, \mathrm{Q}$. et al. Poly[n]catenanes: synthesis of molecular interlocked chains. Science 358, 1434-1439 (2017).

7. Harada, A., Li, J. \& Kamachi, M. The molecular necklace: a rotaxane containing many threaded a-cyclodextrins. Nature 356, 325-327 (1992).

8. Ponnuswamy, N., Cougnon, F. B. L., Clough, J. M., Pantos, G. D. \& Sanders, J. K. M. Discovery of an organic Trefoil knot. Science 338, 783-785 (2012).

9. Kondratuk, D. V. et al. Supramolecular nesting of cyclic polymers. Nat. Chem. 7, 317-322 (2015).

10. Tanaka, T. \& Osuka, A. Conjugated porphyrin arrays: synthesis, properties and applications for functional materials. Chem. Soc. Rev. 44, 943-969 (2015).

11. Kroto, H. W., Heath, J. R., O’Brien, S. C., Curl, R. F. \& Smalley, R. E. C60: buckminsterfullerene. Nature 318, 162-163 (1985).

12. Iijima, S. Helical microtubules of graphitic carbon. Nature 354, 56-58 (1991).

13. Kawasumi, K., Zhang, Q., Segawa, Y., Scott, L. T. \& Itami, K. A grossly warped nanographene and the consequences of multiple odd-membered-ring defects. Nat. Chem. 5, 739-744 (2013).

14. Lin, J. et al. Ultrastable supramolecular self-encapsulated wide-bandgap conjugated polymers for large-area and flexible electroluminescent devices. Adv. Mater. 31, 1804811 (2019).

15. Kistemaker, J. C., Štacko, P., Visser, J. \& Feringa, B. L. Unidirectional rotary motion in achiral molecular motors. Nat. Chem. 7, 890-896 (2015).

16. Baxter, P. N. W., Lehn, J.-M., Fischer, J. \& Youinou, M.-T. Self-assembly and structure of a $3 \times 3$ inorganic grid from nine silver ions and six ligand components. Angew. Chem. Int. Ed. 33, 2284-2287 (1994).

17. Xie, X. et al. Nanogrid arene: a rising nanomolecular integration platform of organic intelligence. Chin. J. Chem. 38, 103-105 (2020).

18. Zhang, G. et al. A robust molecular unit nanogrid servicing as network nodes via molecular installing technology. Mater. Chem. Front. 1, 455-459 (2017).

19. Wei, Y. et al. Organic synthesis of ancient windmill-like window nanogrid at molecular scale. Eur. J. Org. Chem. 2018, 7009-7016 (2018).
20. Yang, L. et al. A novel structure of grid spirofluorene: a new organic semiconductor with low reorganization energy. N. J. Chem. 43, 7790-7796 (2019).

21. Wang, L. et al. Friedel-Crafts bottom-up synthesis of fluorene-based soluble luminescent organic nanogrids. Org. Lett. 16, 1748-1751 (2014).

22. Teator, A. J. \& Leibfarth, F. A. Catalyst-controlled stereoselective cationic polymerization of vinyl ethers. Science 363, 1439-1443 (2019)

23. Yu, M.-N. et al. Emission enhanced and stabilized by stereoisomeric strategy in hierarchical uniform supramolecular framework. Chem 5, 2470-248 (2019).

24. Navarro-Sánchez, J. et al. Peptide metal-organic frameworks for enantioselective separation of chiral drugs. J. Am. Chem. Soc. 139, 4294-4297 (2017).

25. Hoffmann, R. \& Woodward, R. Orbital symmetry control of chemical reactions. Science 167, 825-831 (1970).

26. Houk, K. N. et al. Theory and modeling of stereoselective organic reactions. Science 231, 1108-1117 (1986).

27. Moran, A., Hamilton, A., Bo, C. \& Melchiorre, P. A mechanistic rationale for the 9-Amino(9-deoxy)epi cinchona alkaloids catalyzed asymmetric reactions via iminium ion activation of enones. J. Am. Chem. Soc. 135, 9091-9098 (2013)

28. Kole, G. K., Tan, G. K. \& Vittal, J. J. Anion-controlled stereoselective synthesis of cyclobutane derivatives by solid-state $[2+2]$ cycloaddition reaction of the salts of trans-3-(4-pyridyl) acrylic acid. Org. Lett. 12, 128-131 (2010).

29. Yamada, S. \& Tokugawa, Y. Cation $-\pi$ controlled solid-state photodimerization of 4-azachalcones. J. Am. Chem. Soc. 131, 2098-2099 (2009).

30. Yamada, S. \& Kawamura, C. $[4+4]$ Photodimerization of azaanthracenes in both solution and solid phase controlled by cation $-\pi$ interactions. Org. Lett. 14, 1572-1575 (2012)

31. Li, Y. X. et al. SMART design of a bulk-capped supramolecular segment for the assembly into organic interdigital lipid bilayer-like (ILB) nanosheets. Small 14, 1703151 (2018)

32. Josse, T., De Winter, J., Gerbaux, P. \& Coulembier, O. Cyclic polymers by ring-closure strategies. Angew. Chem. Int. Ed. 55, 13944-13958 (2016).

33. Yu, Y. et al. 4, 5-Diazafluorene-based donor-acceptor small molecules as charge trapping elements for tunable nonvolatile organic transistor. Mem. Adv. Sci. 5, 1800747 (2018)

34. White, P. B. et al. Structurally diverse diazafluorene-ligated palladium(II) complexes and their implications for aerobic oxidation reactions. J. Am. Chem. Soc. 138, 4869-4880 (2016).

35. Lin, D. et al. Carbon cationic relay via superelectrophiles: synthesis of spirodiazafluorenes. Org. Lett. 18, 6220-6223 (2016)

36. Lin, J.-Y. et al. Understanding the molecular gelation processes of heteroatomic conjugated polymers for stable blue polymer light-emitting diodes. J. Mater. Chem. C. 5, 6762-6770 (2017).

37. Feng, Q. et al. Stereoselective photoredox ring-opening polymerization of $\mathrm{O}-$ carboxyanhydrides. Nat. Commun. 9, 1559-1568 (2018).

38. Klumpp, D. A. Superelectrophiles: charge-charge repulsive effects. Chem. Eur. J. 14, 2004-2015 (2008)

39. Yu, L. et al. Proton effects on diazafluorene derivatives with sulfur-rich substituents, a structural, spectroscopic and theoretical study. J. Phys. Chem. A 112, 13672-13678 (2008)

40. Vishweshwar, P., Nangia, A. \& Lynch, V. M. Recurrence of carboxylic acid-pyridine supramolecular synthon in the crystal structures of some pyrazinecarboxylic acids. J. Org. Chem. 67, 556-565 (2002).

41. Yang, Y. et al. Diarylfluorenes-based $\pi$-stacked molecules: synthesis, X-ray crystallography, and supramolecular light-emitting devices. Tetrahedron $\mathbf{6 9}$, 6317-6322 (2013)

42. Plory, P. J. in Principles of Polymer Chemistry 95-102 (Cornell Univ. Press, 1953).

43. Jikei, M. \& Kakimoto, M. Hyperbranched polymers: a promising new class of materials. Prog. Polym. Sci. 26, 1233-1285 (2001).

44. Nagai, M., Huang, J., Zhou, T. \& Huang, W. Effect of molecular weight on conformational characteristics of poly(3-hexyl thiophene). J. Polym. Sci. Part B Polym. Phys. 55, 1273-1277 (2017).

45. Vanhee, S. et al. Synthesis and characterization of rigid rod poly(pphenylenes). Macromolecules 29, 5136-5142 (1996).

46. Naredla, R. R., Zheng, C., Lill, S. O. N. \& Klumpp, D. A. Charge delocalization and enhanced acidity in tricationic superelectrophiles. J. Am. Chem. Soc. 133 13169-13175 (2011)

47. Flory, P. J. The configuration of real polymer chains. J. Chem. Phys. 17, 303-310 (1949)

48. $\mathrm{Hu}$, D. et al. Collapse of stiff conjugated polymers with chemical defects into ordered, cylindrical conformations. Nature 405, 1030-1033 (2000).

49. Tanaka, G. \& Mattice, W. L. Chain collapse by atomistic simulation. Macromolecules 28, 1049-1059 (1995).

50. DeVries, G. A. et al. Divalent metal nanoparticles. Science 315, 358-361 (2007).

51. Yu, J., Wang, Z. \& Chu, B. Kinetic study of coil-to-globule transition. Macromolecules 25, 1618-1620 (1992). 


\section{Acknowledgements}

We thank Xueyan Liu from Shiyanjia Lab (www.Shiyanjia.com) for the COSY and ${ }^{1} \mathrm{H}$ NMR characteristics. We thank Jinwei Liu from Shiyanjia Lab (www.Shiyanjia.com) for the COSY, HRMS, and NOESY characteristics. We thank daxia Xia from Shiyanjia Lab (www.Shiyanjia.com) for the simulation of the vibrational modes. We thank Chenqing from Shiyanjia Lab (www.Shiyanjia.com) for the DLS characteristics. We thank Changjin Ou for single-crystal crystallography analysis. This work was supported by the National Natural Science Foundation of China (21774061, 21602111 and 61935017), National Natural Science Foundation major research program integration project (Grant Number 91833306), the Natural Science Foundation of Jiangsu Province of China (BK20150832), Synergetic Innovation Centre for Organic Electronics and Information Displays, Six Peak Talents Foundation of Jiangsu Province (XCLCXTD-009), and Postgraduate Innovation Fundation of Jiangsu Province (46030CX17747 and SJCX18_0302).

\section{Author contributions}

D.L. has prepared above substrates, performed all of gridizations and polygridizations, analysed all of datum (in terms of stereoselectivity and polymeric characterizations), performed the simulation of chain collapse, and written this manuscript and Supplementary Information. Y.W. has carefully polished up this manuscript and Supplementary Information files, as well as provided crucial insight into the organic synthesis of this work. A.P., He Zhang, C.Z., and X.Z. have performed parts of experiments in terms of the synthesis of substrates. D.L. and Hao Zhang have provided the characterization of PDHG-F samples. L.Y. has provided the theoretical calculation for the geometric optimization of meso-DHG1 and rac-DHG1. Q.F. has provided essential suggestions for this work. L.X. and W.H., both of whom are corresponding authors, have initiated the project, provided enough funds to ensure this work, and supervised every step of the work. Especially, L.X. has created the Drawing Hands pictures.

\section{Competing interests}

The authors declare no competing interests.

\section{Additional information}

Supplementary information is available for this paper at https://doi.org/10.1038/s41467020-15401-x.

Correspondence and requests for materials should be addressed to L.X. or W.H.

Peer review information Nature Communications thanks the anonymous reviewers for their contribution to the peer review of this work. Peer reviewer reports are available.

Reprints and permission information is available at http://www.nature.com/reprints

Publisher's note Springer Nature remains neutral with regard to jurisdictional claims in published maps and institutional affiliations.

\begin{abstract}
cc) (i) Open Access This article is licensed under a Creative Commons Attribution 4.0 International License, which permits use, sharing, adaptation, distribution and reproduction in any medium or format, as long as you give appropriate credit to the original author(s) and the source, provide a link to the Creative Commons license, and indicate if changes were made. The images or other third party material in this article are included in the article's Creative Commons license, unless indicated otherwise in a credit line to the material. If material is not included in the article's Creative Commons license and your intended use is not permitted by statutory regulation or exceeds the permitted use, you will need to obtain permission directly from the copyright holder. To view a copy of this license, visit http://creativecommons.org/ licenses/by/4.0/.
\end{abstract}

(C) The Author(s) 2020 